Classification

Physics Abstracts

07.05.Tp $-47.55 . \mathrm{Mh}-81.05 . \mathrm{Rm}$

\title{
Influence of the Topology on the Flow Properties of Porous Media Explored from 3D Simulations
}

\author{
Patricia Jouannot and Jean-Paul Jernot \\ LERMAT, URA CNRS 1317, ISMRA, 14050 Caen Cedex, France
}

\begin{abstract}
Résumé. - Une simulation tridimensionnelle d'empilement aléatoire de sphères, digitalisée sur une trame CFC, est densifiée au moyen de dilatations ou fermetures. En utilisant séparément ces transformations, la densification de l'empilement initial suit deux chemins topologiques distincts. Des écoulements sont simulés par des dilatations géodésiques dans les réseaux poreux de chacune des structures. Pour une valeur donnée de la porosité, les résultats obtenus pour l'écoulement mettent en évidence l'influence prépondérante des propriétés topologiques.
\end{abstract}

\begin{abstract}
A 3D-simulated random packing of spheres is digitized and densified with two basic tools of mathematical morphology: dilation and closing. Using these transformations separately, the densification of the initial structure follows two different topological paths. As a consequence, a fluid flow simulated by geodesic dilations inside the porous networks shows two different behaviours depending on the topological paths of the densification.
\end{abstract}

\section{Simulated Structures}

A 3D random packing of monosized spheres is simulated on a SUN computer. The stacking is then progressively densified with two classical tools of mathematical morphology: dilation and closing.

InItial StRUCTURE. - The starting point of the simulation is a random packing of spheres generated from a FORTRAN program from Visscher and Bolsterli [1]. A small part of the stacking is digitized on a Face-Centred Cubic grid [2] leading to a simulation box containing 184 planes of size $151 \times 174$. The morphological transformations are performed on this image but the measurements are made on a smaller image of size $131 \times 154 \times 164$ to avoid the border effects of the transformations. This reduced box contains about 240 spheres.

Densification of the Stacking. - Two basic tools of mathematical morphology are used to densify the initial structure: dilation and closing [3]. These transformations are applied in 3D 

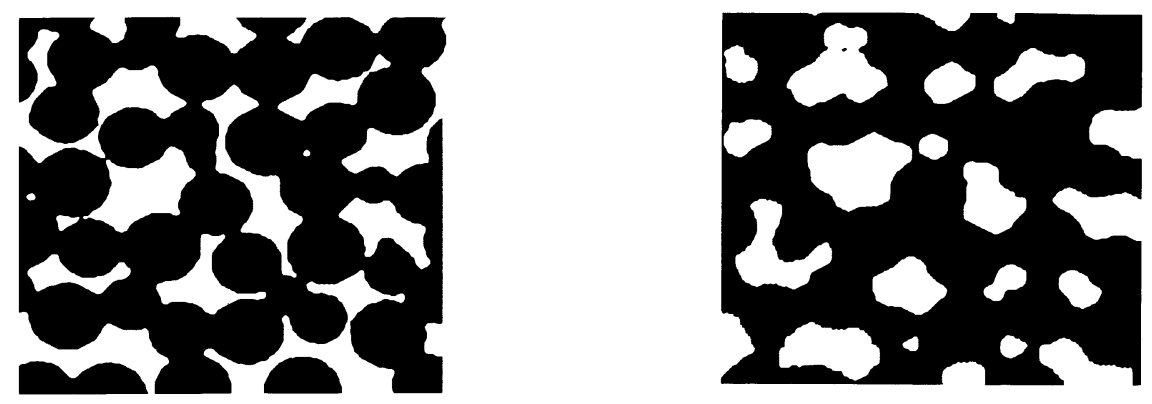

Fig. 1. - Plane cuts through the dilated structure D1 (left) and the closed structure F3 (right).

space on the initial structure digitized on the FCC grid. In this case, the elementary structuring element, B, is a cuboctahedron (one central point with its twelve equidistant neighbours). Successive dilations or closings carried out on the initial structure give the $3 \mathrm{D}$ dilated and closed images. The dilated structures (respectively closed) named $\mathrm{D} i(\mathrm{~F} i)$ are the result of a transformation of size $i$ realized on the initial structure D0 (F0, identical to D0) using the cuboctahedron. An example of these structures is given in Figure 1 where plane cuts through the structures D1 and F3 are visualized.

The topology of each structure is quantitatively described by the Euler-Poincaré characteristic, $N_{3}$, measured in 3D space. This parameter is defined by

$$
N_{3}=s-\sum_{s} G
$$

with: $s$, the number of disconnected surfaces, $G$, the genus of each surface (the genus of a surface being the maximum number of cuts that can be made through this surface without disconnecting it in several parts) [4].

In the case of a local analysis, the value of $N_{3}$ must be referred to a unit volume of structure $\left(N_{\mathrm{V}}=N_{3} / V\right)$. For all the simulations, the field size is constant and is chosen as the unit volume. This parameter is followed as a function of the densification for dilated and closed structures. At the beginning of the densification, there are no major changes in the topological state of the stacking (D0, D1, F0, F1). The slight decrease of $N_{\mathrm{V}}$ is only due to the increase of the compacity [2]. A further densification induces a modification of the porous network: some branches are removed, the genus decreases and $N_{\mathrm{V}}$ increases (D2, D3, F2, F3, F4). Then, the pores become isolated (D4, D5 , .., F5 ...) and finally disappear $\left(N_{\mathrm{V}}=0\right)$. The two processes of densification can be compared in Figure 2. For the same porosity, two different topological states are obtained. Thus, two topological paths are followed all along the densification process, different connectivity properties being associated with each path.

\section{Geodesic Propagations Through the Porous Networks}

The topology of the structures has just been described by the global parameter $N_{\mathrm{V}}$. More information can be obtained from an exploration of the porous network taking into account its local connectivity.

Exploration of The Porous Phase. - The different porous networks are explored by a simple but powerful transformation of mathematical morphology: the geodesic dilation [5]. 

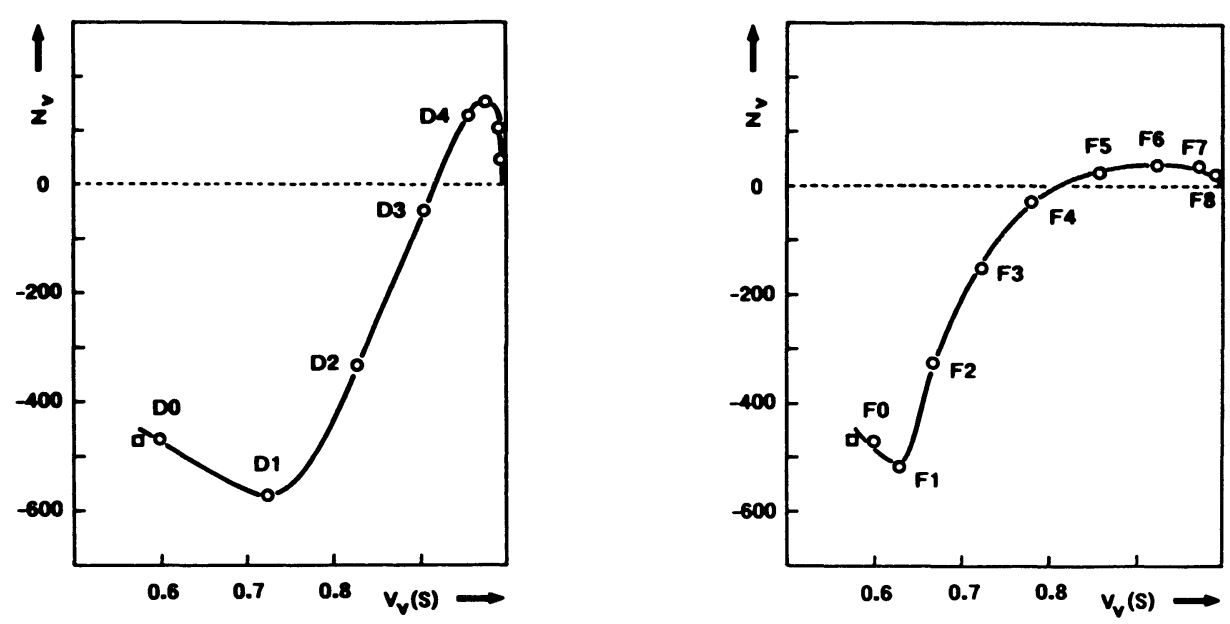

Fig. 2. - Euler-Poincaré characteristic per unit volume, $N_{\mathrm{V}}$, as a function of the compacity, $V_{\mathrm{V}}(\mathrm{S})$, for the dilated $(\mathrm{D} i)$ and closed $(\mathrm{F} i)$ structures.

For each structure, the porous phase of the upper plane is taken as a marker and the geodesic dilations start from this plane. A kind of flow is generated from the upper plane $(z=0)$ to the lower one $(z=163)$. Each elementary step of the propagation is called the time, $t$. The progressive invasion of the porous phase is described by two parameters:

- the percentage of the porous surface invaded on the lower plane, \% $\mathrm{A}(\mathrm{P})$;

- the percentage of the porous volume invaded in the whole structure, $\% \mathrm{~V}(\mathrm{P})$.

The curves corresponding to the structures D1 and F3 are given in Figure 3. Following the topological evolution of the structures (Fig. 2), four steps can be distinguished according to the differences of behaviours with respect to the propagation:

i) A complete filling of the porous phase is obtained for the initial structures D0, D1, F1. The time necessary to reach the bottom plane or to invade the whole volume is nearly the minimum one (163). This is due to an almost plane filling of the porous phase and a regular invasion illustrated by the linear variation of the porous volume invaded with the propagation time.

ii) For the structures D2 and F2, a still complete filling of the pores is observed: the porous network is thus entirely interconnected. Nevertheless, the propagation time is longer than for the initial structures, the paths being more and more tortuous.

iii) From now on (D3, F3, F4) some parts of the porous network become isolated. A whole filling of the porous phase is no more possible. The partial invasion of the bottom plane is irregular and the invasion time is very long.

iv) For the last structures, the propagation flow does not reach the bottom plane. The porous phase contains a large proportion of isolated pores and the invaded volume is small.

Simulation of FlOW. - The exploration of the porous network by means of geodesic propagations looks like a simulation of flow $[6,7]$. The volumetric rate of flow per unit volume 

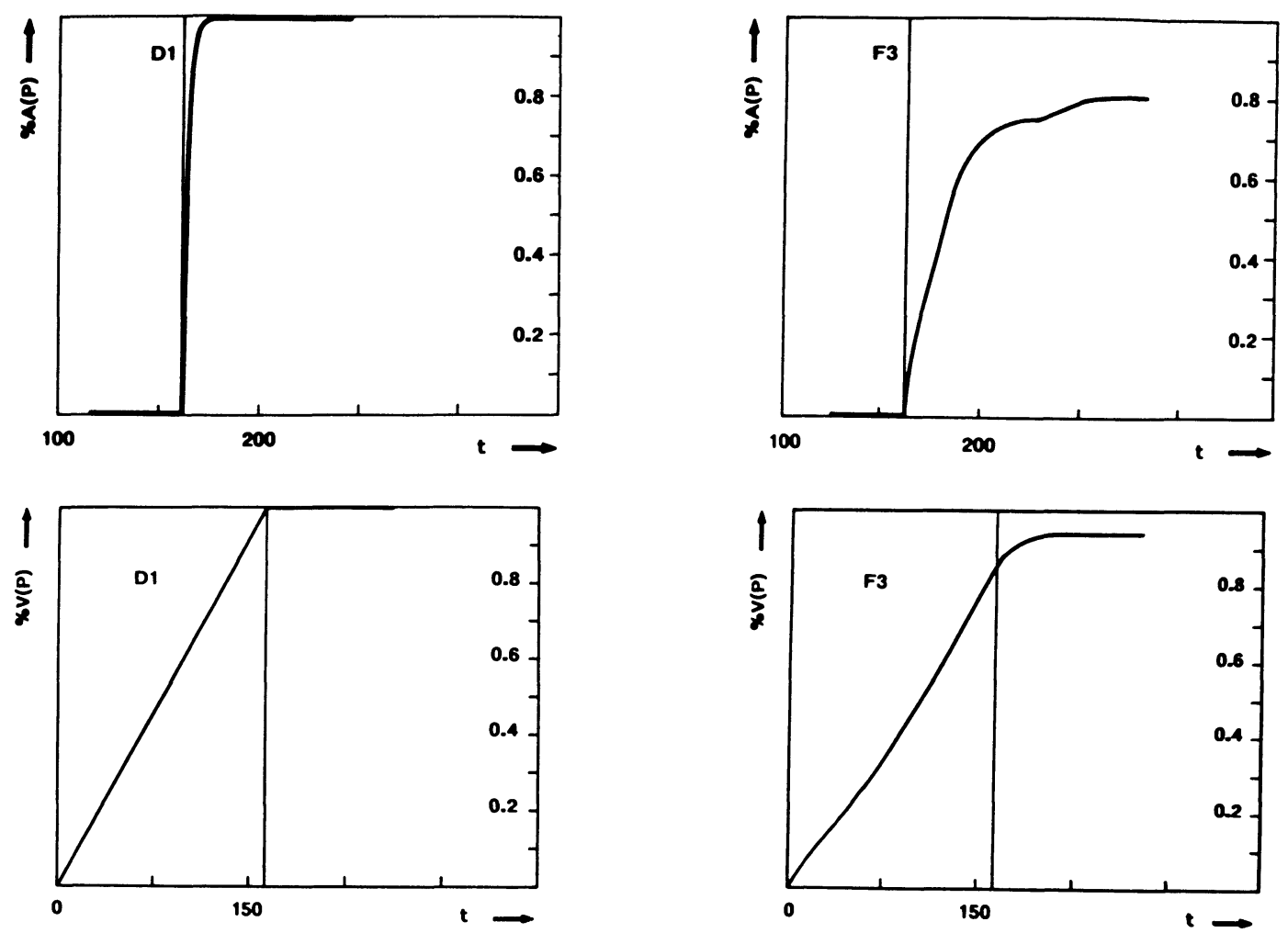

Fig. 3. - Area fraction of the porous phase invaded in the exit plane, $\% \mathrm{~A}(\mathrm{P})$, and volume fraction of the porous phase invaded inside the whole field, $\% \mathrm{~V}(\mathrm{P})$, as a function of the propagation time, $t$, for the $\mathrm{D} 1$ and F3 structures.

Table I. - Evolution of flow for the dilated and closed structures (F0, identical to D0, has not been reported for the closed structures). $V_{V}(P)$ is the volumic fraction of the porous phase and $Q_{V}$ the volumetric rate of flow per unit volume of structure.

Dilated structures

\begin{tabular}{|c|c|c|c|c|}
\hline Structure & D0 & D1 & D2 & D3 \\
\hline$V_{\mathrm{V}}(\mathrm{P})$ & 0.398 & 0.275 & 0.170 & 0.092 \\
$Q_{\mathrm{V}}$ & 0.398 & 0.271 & 0.153 & 0.053 \\
\hline
\end{tabular}

\section{Closed structures}

\begin{tabular}{|c|c|c|c|c|}
\hline Structure & F1 & F2 & F3 & F4 \\
\hline$V_{\mathrm{V}}(\mathrm{P})$ & 0.373 & 0.334 & 0.278 & 0.215 \\
$Q_{\mathrm{V}}$ & 0.373 & 0.323 & 0.219 & 0.116 \\
\hline
\end{tabular}

of structure, $Q_{\mathrm{V}}$, can be defined as the porous volume invaded during the time interval $\Delta t$ :

$$
Q_{\mathrm{V}} \cong \Delta V_{\mathrm{V}} / \Delta t
$$

The evolution of this parameter as a function of the densification is reported in Figure 4 and the results are collected in Table I (for simplification, $\Delta t=100$ steps is taken as the unit time). In the figure, the dotted line represents a linear decrease of $Q_{\mathrm{V}}$ with the compacity. The results concerning D0 (F0), D1 and F1 are placed on this dotted line. For these structures, the value of $N_{3}$ reported per unit volume of the solid phase, i.e. $N_{3} / V(\mathrm{~S})=N_{\mathrm{V}} / V_{\mathrm{V}}(\mathrm{S})$, is almost the same. This means that they are topologically equivalent and the flow only depends on the quantity of 

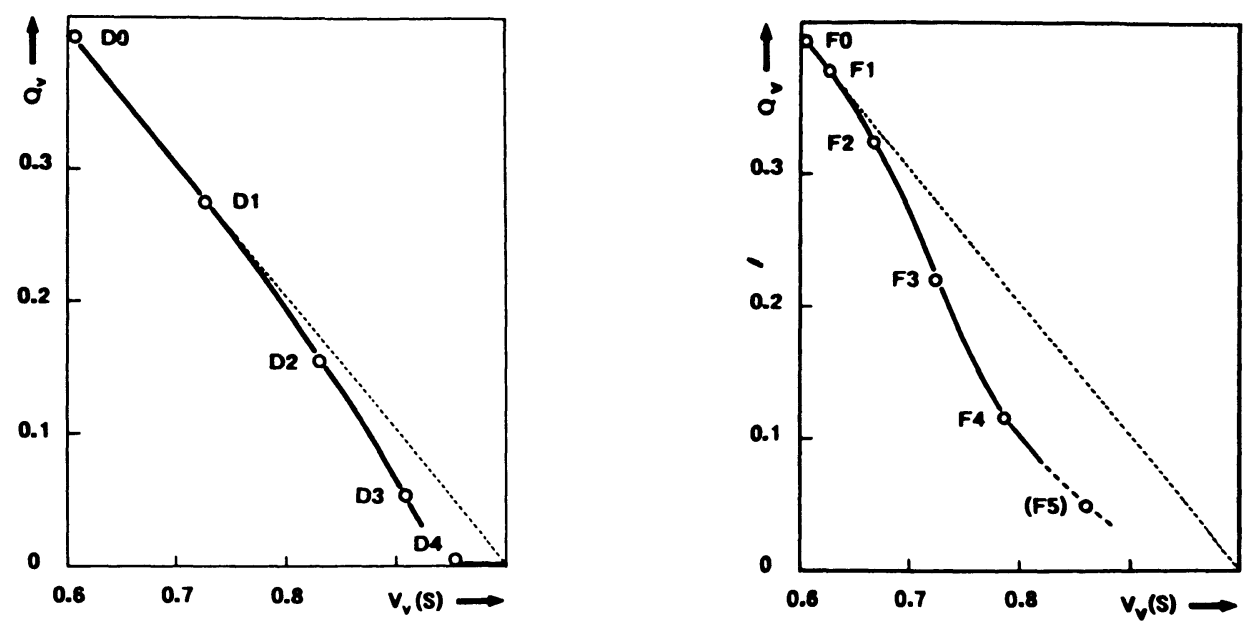

Fig. 4. - Flow per unit volume, $Q_{\mathrm{v}}$, as a function of the densification for the dilated (D $\left.i\right)$ and closed (Fi) structures.

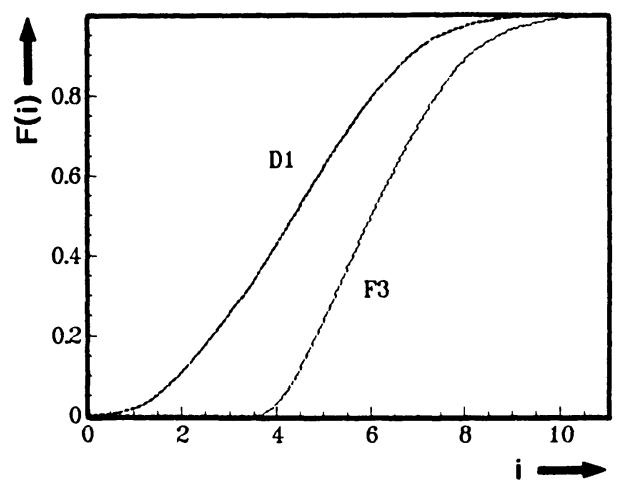

Fig. 5. - Size distribution curves obtained by 3D openings of the porous networks for the D1 and F3 structures. $\mathrm{O}_{i}(\mathrm{P})$ being the porous set after one opening of size $i, F(i)$ is defined as $F(i)=1-V_{\mathrm{V}}\left[\mathrm{O}_{i}(\mathrm{P})\right] / V_{\mathrm{V}}\left[\mathrm{O}_{0}(\mathrm{P})\right]$.

the porous phase. For the more densified structures, the linear behaviour is no longer observed: topological changes occur inside the porous networks. They take place at lower densities for the structures $\mathrm{F} i$ than for the structures $\mathrm{D} i$ (Fig. 2) and $Q_{\mathrm{V}}$ decreases more rapidly for the closed structures than for the dilated ones. However, the decrease of $Q_{\mathrm{V}}$ could also be attributed to a decrease of the size of the pores along the densification. Then, for each structure (Di, Fi), a granulometry of the porous phase has been carried out by openings [8] in 3D space.

For comparable values of $N_{\mathrm{V}}$ (e.g. D2 and F2), as expected, the corresponding results of $Q_{\mathrm{V}}$ are higher for the closed structure F2 possessing a larger mean size of pores and a larger porosity.

For comparable values of the porosity (e.g. D1 and F3), the mean pore size is larger for the porous network of F3 than for D1 (Fig. 5). With almost the same porosity and a mean pore size roughly $50 \%$ larger for F3 than for D1, one can expect that the flow would be easier through the F3 structure. Surprisingly, the reverse is observed: the value of $Q_{\mathrm{V}}$ is lower for F3 than for D1. This indicates that, in this case, the flow is more influenced by the topology of the porous network than by its size. 


\section{Conclusion}

Two transformations of mathematical morphology, dilation and closing, have been used to densify a random stacking of spheres. The resulting structures follow two different topological paths: the dilation promotes the densification while the closing favours the topological modifications of the porous medium.

The two topological paths of densification induce two different behaviours with respect to the same simulation of flow. At the beginning, the flow only depends on the porosity because the topological characteristics are equivalent. Then, for comparable values of the porosity, the flow is mainly disrupted by the topological modifications of the porous network, whatever the pore size. This emphasizes qualitatively the role played by the topology on the flow properties of a porous medium.

\section{Acknowledgements}

This work was performed in the frame of the "Pôle Traitement et Analyse d'Image" de BasseNormandie.

\section{References}

[1] Visscher W.M. and Bolsterli M., Random packing of equal and unequal spheres in two and three dimensions, Nature 239 (1972) 504-507.

[2] Bhanu Prasad P. and Jernot J.P., Topological description of the densification of a granular medium, $J$. Microsc. 163 (1991) 211-220.

[3] Serra J., Image Analysis and Mathematical Morphology, Vol. 1 (London Academic Press, 1982).

[4] DeHoff R.T., Aigeltinger E.H. and Craig K.R., Experimental determination of the topological properties of three dimensional microstructures, J. Microsc. 95 (1972) 69-91.

[5] Lantuéjoul C. and Beucher S., On the use of the geodesic metric in image analysis, J. Microsc. 121 (1981) 39-49.

[6] Jernot J.P., Bhanu Prasad P. and Demaleprade P., Three-dimensional simulation of flow through a porous medium, J. Microsc. 167 (1992) 9-21.

[7] Jouannot P., Étude topologique de structures aléatoires biphasées, Thèse de Doctorat de l' Université de Caen (1994).

[8] Matheron G., Éléments pour une théorie des milieux poreux, (Masson, Paris, 1967). 